\title{
Modelagem das mudanças espaço-temporais de áreas úmidas: estudo de caso da Região Administrativa de Abitibi-Témiscamingue, Québec, Canadá*
}

\author{
Spatial modeling of changes in open wetlands by cellular \\ automata: a case study on the administrative region of \\ Abitibi-Témiscamingue, Quebec, Canada
}

\author{
Mariana de Oliveira Tiné^ \\ Liliana Perez \\ Roberto Molowny-Horas ${ }^{\bullet}$
}

Recebido 30 de agosto de 2019; aceito 04 de novembro de 2019

\section{Resumo}

As áreas úmidas estão entre os ecossistemas mais produtivos que existem, elas abrigam uma ampla biodiversidade de aves, peixes, vegetais, entre outros. Elas também desempenham papel fundamental no que diz respeito à mitigação e adaptação climática. Apesar de sua grande importância, estes ecossistemas estão cada vez mais ameaçados, seja pela interferência humana, seja pelas mudanças climáticas. Cerca de $35 \%$ das áreas úmidas do planeta se localizam no Canadá, onde grande parte está na zona de floresta boreal. Este estudo tem como objetivo simular as mudanças

* Artigo baseado em dissertação de mestrado "Modélisation spatiale des changements dans les milieux humides ouverts par automate cellulaire: étude de cas sur la région administrative de l'Abitibi-Témiscamingue, au Québec", apresentados em abril de 2018 e os resultados publicados em International Journal of Applied Earth Observation and Geoinformation.

- MRC du Haut-Saint-Laurent, Canadá, correio eletrônico: marianatine@gmail.com. ORCID: https://orcid.org/0000-0001-7101-1194.

- Université de Montréal, Canadá, correio eletrônico:1.perez@umontreal.ca. ORCID: https://orcid.org/0000-0002-6599-9893.

- CREAF, Espanha, correio eletrônico: roberto@creaf.uab.cat. ORCID: https://orcid.org/0000-0003-2626-6379. 
espaço-temporais destes ecossistemas através de técnicas de modelagem híbrida. Para isto, foram usadas imagens de cobertura da terra multitemporais, interpretadas a partir do satélite LANDSAT, com as quais foi possível simular cenários até o ano de 2055, cujos resultados foram validados através de técnicas de comparação de mapas. A análise de mudanças mostrou um aumento das áreas úmidas de cerca de $63 \%$ entre os anos de 1985 e 2005, tendência que persistiu até 2055, com um aumento contínuo destas na região de estudo. A análise e simulação de cenários futuros pode ajudar e apoiar a gestão e planejamento no que diz respeito à conservação destes ecossistemas tão importantes.

Palavras chaves: sensoriamento remoto, Landsat, modelagem espacial, modelos hibridos, sistemas complexos.

\begin{abstract}
Wetlands are among the most productive ecosystems that exist, they have a wide biodiversity of birds, fish, vegetables, among others. They also play a key role in climate mitigation and adaptation. Despite their great importance, these ecosystems are increasingly threatened, either by human interference or by climate change. About $35 \%$ of the planet's wetlands are located in Canada, where much of it is in the boreal forest zone. The aim of this work is to simulate the spatio-temporal changes of these ecosystems using a hybrid model. For this purpose, multitemporal land cover images were interpreted from the LANDSAT satellite, which it was possible to simulate scenarios until the year 2055. The results were validated using map-comparison techniques. The analysis of changes showed an increase in wetlands about $63 \%$ between 1985 and 2005, persisting into 2055 with a continuous increase of these areas in the region. Analysis and simulation of future scenarios can support management and planning related to the conservation of these important ecosystems.

Key words: remote sensing, Landsat, spatiotemporal modelling, wetlands, hybrid model, complex systems.
\end{abstract}

\title{
Introdução
}

As áreas úmidas são algumas das zonas de biodiversidade mais importantes do mundo, elas são ecossistemas complexos, únicos e produtivos, possuindo maior diversidade de espécies e reciclagem de nutrientes do que a maioria dos outros ecossistemas (Menard et al., 2013; Poulin et al., 2016). As estimativas globais da superfície destas áreas variam de 5,3 a 12,8 milhões de $\mathrm{km}^{2}$. No entanto, as áreas úmidas estão experimentando um rápido declínio, os pesquisadores estimam que mais de 50\% destas desapareceram em todo o mundo (Zedler and Kercher, 2005).

As áreas úmidas correspondem a aproximadamente $13 \%$ do território canadense, onde 25\% ocorre na Zona Boreal (Environment and Climate Change Canada, 2016; 
Menard et al., 2013). As áreas úmidas do Canadá desempenham um papel importante no balanço global de carbono porque têm cerca de 150 milhões de toneladas destes acumuladas nas turfeiras, o que é 25 vezes a quantidade do combustível fóssil liberado anualmente no mundo (Efron, 1988). Como essas áreas possuem águas rasas, os episódios de secas severas e de temperaturas mais altas causariam maior evaporação, facilitando o aumento da emissão de carbono na atmosfera, o que afetaria significativamente o clima em escala global (Frolking et al., 2011).

Deve-se presumir que as áreas úmidas experimentarão grandes variações de extensão quando enfrentarem cenários climáticos futuros que prevejam tanto invernos mais curtos quanto verões mais longos. Estas variações não influenciarão apenas o clima terrestre, mas também todas as espécies que nele habitam. Alguns estudos destacaram que no extremo sul do Canadá o desaparecimento de áreas úmidas pode colocar algumas populações à beira da extinção, principalmente devido à contínua mudança no uso da cobertura da terra (Eamer et al., 2010).

Em resposta aos impactos que a mudança climática pode trazer para os ecossistemas, os pesquisadores recorreram ao uso de modelos computacionais que permitem estudar e entender melhor a dinâmica temporal e espacial observada em áreas úmidas (Arsanjani et al., 2015; Gong et al., 2014; Hattermann et al., 2008; Yu et al., 2010). Eles podem ser usados para analisar mudanças observadas, entender seus elementos e, em seguida, prever cenários futuros. As complexidades destas mudanças podem ser modeladas e simuladas pela integração de abordagens metodológicas de sensoriamento remoto (SR), sistemas de informação geográfica (SIG) e modelagem de sistemas complexos (MSC) (Batty, 2012). Modelos combinando múltiplas abordagens como autômatos celulares (AC), redes neurais artificiais (RNAs), cadeia Markov (CM), regressões logísticas (RL) e programação multi-objetivo, por exemplo, estão sendo cada vez mais utilizados para modelar sistemas ambientais (Arsanjani et al., 2015; Chen et al., 2008; Hyandye and Martz, 2017; Luo et al., 2015; Qiang and Lam, 2015).

Este estudo teve como objetivo desenvolver um modelo computacional para simular e estudar as mudanças nas áreas úmidas abertas da região de AbitibiTémiscamingue, no Québec, Canadá. Para isto, foram seguidas as seguintes etapas:

1. Reclassificação de imagens LANDSAT de 1985, 1995 e 2005, e avaliação das mudanças e tendências em áreas úmidas abertas durante esse período;

2. Elaboração de mapas de transição potencial da cobertura de terra desenvolvidos com o auxílio de regressão logística para simular mudanças em áreas úmidas abertas;

3. Elaboração de uma abordagem de modelagem híbrida afim de simular mudanças das áreas úmidas abertas até 2055. 


\section{Materiais e métodos}

\section{Area de estudo e caracterização das áreas úmidas}

A região administrativa de Abitibi-Témiscamingue está localizada no extremo oeste da provincia de Québec. É uma das mais vastas regiões da província com uma superfície de $64656 \mathrm{~km}^{2}$ (Figura 1). Ela se caracteriza por ser uma zona de transição entre a floresta mista temperada (ao sul) e a floresta boreal (ao norte) e possui um relevo plano, com a presença de baixas colinas principalmente na região nordeste. Estas características favorecem o desenvolvimento de planos hídricos, e consequentemente de áreas úmidas. Possui um clima frio e seco, com verões frios (Kottek et al., 2006). É uma região de baixa densidade demográfica, com um total de 147 mil habitantes em 2013, onde 50\% destes estavam situados em apenas 5 municípios (de um total de 79).

As áreas úmidas são ecossistemas complexos que podem ser encontrados em todo o planeta. Entretanto, devido às diferenças entre os regimes hidráulicos e climáticos regionais, existe uma grande variedade entre elas. Da mesma forma, a definição destas áreas, bem como a sua classificação, variam por todo o mundo (Ménard et al., 2007). De acordo com a Convenção de Ramsar, as áreas úmidas são: "áreas de pântano, charco, turfa ou água, natural ou artificial, permanente ou temporária, com água estagnada ou corrente, doce, salobra ou salgada, incluindo áreas de água marítima com menos de seis metros de profundidade na maré baixa" (Ramsar, 2010).

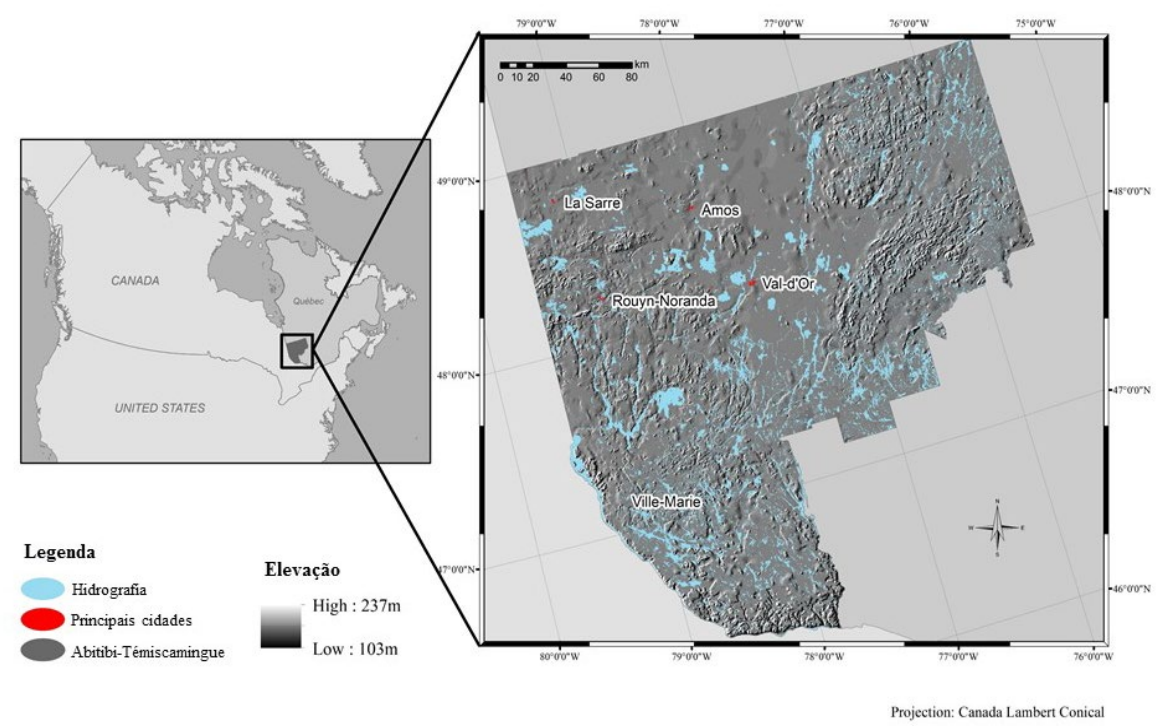

Figura 1. Localização da região administrativa de Abitibi-Témiscamingue. 
No Canadá, o Grupo Nacional de Trabalho sobre as Terras Úmidas (GTNTH) define estas áreas como: "uma terra saturada de agua durante um período suficientemente longo para que existam processos que se caracterizam por uma fraca drenagem do solo e diferentes tipos de atividades biológicas adaptadas a estes meios" (Warner \& Rubec, 1997).

Para este estudo foram utilizadas as áreas úmidas visíveis nas imagens de satélite, aqui denominadas "áreas úmidas abertas", ou seja, aquelas onde a cobertura vegetal é $<25 \%$ (Lemelin et al., 2010). Estas áreas correspondem a 13,3\% do territorio da região de estudo (Lachance et al., 2016).

\section{Preparação dos dados de cobertura da terra}

Os dados de entrada do modelo foram extraídos de três imagens LANDSAT TM (1985, 1995 e 2005), provenientes do estudo de Valeria et al. (2014), que foram processadas e transformadas para corresponder aos interesses do estudo. Foi realizada uma reclassificação (generelização) da cobertura da terra e também da resolução espacial, que passou de 30×30 para 90×90 m, afim de agilizar o processo de modelagem. A reclassificação das imagens foi realizada através do método algorítimico Maximum Likelihood Classifier (MLC). As coberturas da classificação inicial foram generalizadas, por exemplo, as 3 classes correspondentes às áreas úmidas se transformaram em apenas uma classe. Ao final da reclassificação restaram 5 classes: água, floresta, zona urbana, agricutura e áreas úmidas abertas, que foram utilizadas no modelo.

\section{Modelo Híbrido}

Cada técnica de modelagem de sistemas complexos, como é o caso das áreas úmidas, possui pontos fortes e limitações, tornando sua adequação para modelagem espaçotemporal específica de cada problema. Dessa forma, os modelos híbridos emergiram da necessidade de integrar duas ou mais técnicas, possibilitando a junção de forças e a superação de fragilidades a fim de fazer previsões mais precisas das mudanças de cobertura (Chen et al., 2008; Hyandye and Martz, 2017). No presente estudo, as técnicas de regressão logística (RL), cadeia Markov (CM) e autômatos celulares (AC) foram integradas em um único modelo.

\section{Regressão Logística}

A regressão logística é uma abordagem amplamente utilizada para a modelagem preditiva do uso da terra (Arsanjani et al., 2012; Feng et al., 2016; Mas et al., 2014). No presente estudo modelou-se a probabilidade de mudança de uma única classe de cobertura terrestre para outra, dentro de um intervalo de tempo pré-definido, assumindo uma resposta binomial (0/1, ou seja, sem mudança / com mudança) cuja 
probabilidade foi determinada por uma função logística. Por sua vez, um conjunto cuidadosamente escolhido de variáveis preditivas contínuas foi usado para avaliar essa probabilidade de mudança em cada classe de cobertura da terra. O procedimento de RL consistiu em maximizar o logarítmo de uma probabilidade binomial L como:

$$
\log \mathcal{L}(y=1 \mid X)=\sum_{i}\left(y_{i} \cdot \log p_{i}-\left(1-y_{i}\right) \cdot \log \left(1-p_{i}\right)\right)
$$

Onde $X$ é uma matriz com linhas e colunas representando as observações (isto é, cobre classes de interesse em várias localizações espaciais) e as variáveis preditivas respectivamente, e $y_{i}$ é a resposta $(0 / 1)$ da observação $i$. Além disso, $p_{i}$ é a probabilidade da variável de resposta $y_{i}=1$ para a observação $i$ e é especificada através de uma função logística:

$$
p_{i}\left(y_{i}=1\right)=\frac{e^{\beta \cdot x_{i}}}{1+e^{\beta \cdot x_{i}}}
$$

A função $p_{i}$ representa a probabilidade de uma resposta binária e $\beta$ é um vetor de linha contendo os parâmetros de regressão desconhecidos. A maximização do $\log \mathcal{L}$ foi realizada variando os parâmetros $\beta$ até que $\log \mathcal{L}$ não pudesse ser mais otimizado. A abordagem de RL forneceu um submodelo que gerou a probabilidade de mudança para uma única transição entre duas classes de cobertura da terra. O resultado da técnica de RL foi um conjunto de mapas de transição potencial que indicavam o grau de probabilidade para que uma determinada transição ocorresse em cada pixel. Os mapas de transição potencial foram então dados como entradas para o algoritmo AC / cadeia Markov (Eastman, 2015; Mas et al., 2014).

\section{Autômatos celulares / Cadeia Markov}

O conceito de autômatos celulares foi desenvolvido pelos matemáticos Alan Turing e John Von Neumann na década de 1940 (Langlois, 2008). Os modelos de AC têm a capacidade de representar sistemas complexos com base em regras simples e lógicas. São estruturas discretas e dinâmicas que representam um espaço e possuem quatro elementos básicos: células (chamadas de pixels neste estudo), estados, vizinhança e regras. Pixels são as unidades espaciais e têm um valor atribuído a eles, que são os estados. Por sua vez, os estados têm um valor em cada etapa de tempo, o que determinará as relações entre um pixel e os pixels vizinhos, formando a vizinhança (Chen et al., 2008). As regras determinarão os estados de cada pixel na próxima etapa de tempo (Kumar et al., 2016).

Uma matriz de transição da cadeia Markov dá a área total (em pixels) que muda entre quaisquer duas classes de cobertura da terra em um dado intervalo de tempo. 
Ao contrário da técnica dos $\mathrm{AC}$, essas matrizes de transição Markov não fornecem informações sobre a influência das células vizinhas (Eastman, 2015). O modelo usado neste estudo faz uso dos AC para explicar a contigüidade espacial na modelagem de transições de cobertura terrestre. Por sua vez, a quantidade de área que muda de uma classe para outra, em cada etapa de tempo, é definida por uma matriz de transição Markov calculada a partir das mesmas duas imagens que foram usadas para determinar os mapas de transição potencial.

\section{Procedimento de Modelagem}

$\mathrm{Na}$ primeira etapa da modelagem as transições mais importantes entre as classes de cobertura da terra foram identificadas entre o tempo 1 (1985) e o tempo 2 (1995). Para simplificar a análise, todas as mudanças ocorrendo entre os conjuntos de cobertura com menos de 10 ha foram ignoradas, porque foi notado que as maiores mudanças estavam acima dessa marca. Finalmente, apenas 15 tipos de transições da terra foram geradas, todas as outras transições foram descartadas.

Na segunda etapa, um total de 6 variáveis preditivas foram preparadas e utilizadas pelo algoritmo da RL para gerar os mapas de transição potencial. A primeira variável consistia em distâncias de estradas, definidas como a distância euclidiana entre cada pixel em 1985 e o pixel da estrada mais próxima. As outras cinco variáveis correspondiam ao número total de pixels pertencente à mesma classe do pixel central dentro de um raio quadrado de $7 \times 7$ pixels no mapa de cobertura de 1985 . Ou seja, resumiu-se em cada pixel a quantidade de pixels adjacentes que pertenciam a cada uma das cinco categorias, gerando assim 5 mapas (um para cada tipo de cobertura), desta forma a vizinhança de cada pixel foi determinada.

$\mathrm{Na}$ terceira etapa, para cada transição válida, calculou-se mapas de transição potencial baseados na RL. Para determinar os coeficientes de regressão, usou-se as variáveis preditivas mencionadas acima e as 15 transições de classe relevantes entre os mapas de 1985 e 1995 como variável de resposta (ou seja, variável $y_{i}$ na Eq. 1). Os 15 mapas de transição potencial resultantes da análise foram agrupados em um conjunto de 15 submodelos de transição. A validação de transição potencial da RL foi realizada antes de prosseguir para a próxima etapa.

$\mathrm{Na}$ quarta etapa, esses submodelos foram usados para realizar as projeções do mapa de cobertura da Cadeia Markov. Esses mapas foram simulados para os anos de 2015, 2025, 2035, 2045 e 2055, a partir do mapa de cobertura da terra gerado para o ano de 2005.

Finalmente, na quinta e última etapa, a validação entre projeções e observações foi realizada comparando o mapa de cobertura da terra projetado para 2005 com o mapa real de 2005. 


\section{Validação}

Uma validação rigorosa dos resultados projetados é uma etapa fundamental para garantir a precisão e exatidão de um procedimento de modelagem. Neste estudo foram validados tanto os resultados da RL como os mapas de cobertura da terra gerados. Para os resultados de RL foi utilizado o método ROC (Receiver Operating Characteristic), cujo valor de acima de 0,7 é considerado bom, enquanto valores acima de 0,9 são considerados excelentes, pois indica o grau de ajuste do modelo (Lin et al., 2011). A precisão dos resultados foi avaliada comparando a imagem da cobertura da terra projetada com o mapa existente.

O processo de validação deve avaliar a capacidade do modelo em produzir com precisão quantidades e localização de classes de células em um mapa (Pontius, 2000). Foi utilizada a metodologia de validação proposta por Pontius (2000) e Pontius e Millones (2011) na qual a concordância entre dois mapas é calculada em termos do número de células em cada categoria $\left(\mathrm{K}_{\mathrm{no}}\right)$ e da localização espacial das células em cada categoria $\left(\mathrm{K}_{\text {location}}\right)$. Também foram calculadas estatísticas relacionadas à concordância e discordância (QD e $\mathrm{AD}$ ) entre os mapas proposta por Pontius e Millones (2011).

\section{Resultados}

Quanto à validação dos resultados dos submodelos gerados à partir da RL, o índice ROC variou de 0,68 a 0,99 , com um valor médio de 0,84 . No que se refere aos índices Kappa, o $\mathrm{K}_{\text {no }}$ mostra um valor muito alto $(0,9584)$, indicando que a simulação especificou a quantidade (ou seja, o número de pixels em cada classe nos mapas real e simulado) corretamente. $O$ valor relativamente alto do índice $K_{\text {location }}(0,8042)$ mostra que a localização especificada da simulação (ou seja, pixels da mesma classe são encontrados na mesma localização nos mapas real e simulado) foi satisfatória. Finalmente, os valores muito baixos dos índices QD $(0,0029)$ e AD $(0,0248)$ também confirmaram que o mapa de cobertura simulado concordou bem com o real em quantidade e alocação, respectivamente.

Após a validação do modelo, foram projetados cinco mapas de cobertura da terra, cada um correspondendo a uma década (o modelo é executado em intervalos de tempo correspondentes a dez anos), de 2015 a 2055. Para entender os resultados em termos de dinâmica espaço-temporal anterior de áreas úmidas na região, calculamos

a extensão total destas a partir dos dados de sensoriamento remoto. O padrão observado de expansão capturado pelas imagens LANDSAT indicou um aumento na cobertura de áreas úmidas de mais de 60\% em 2005 em comparação com a área mapeada em 1985. Da mesma forma, os resultados de simulação baseados na dinâmica espacial durante as duas décadas mostraram um crescimento progressivo em toda a região. O mapa de simulado para o ano de 2055 mostra um aumento de 


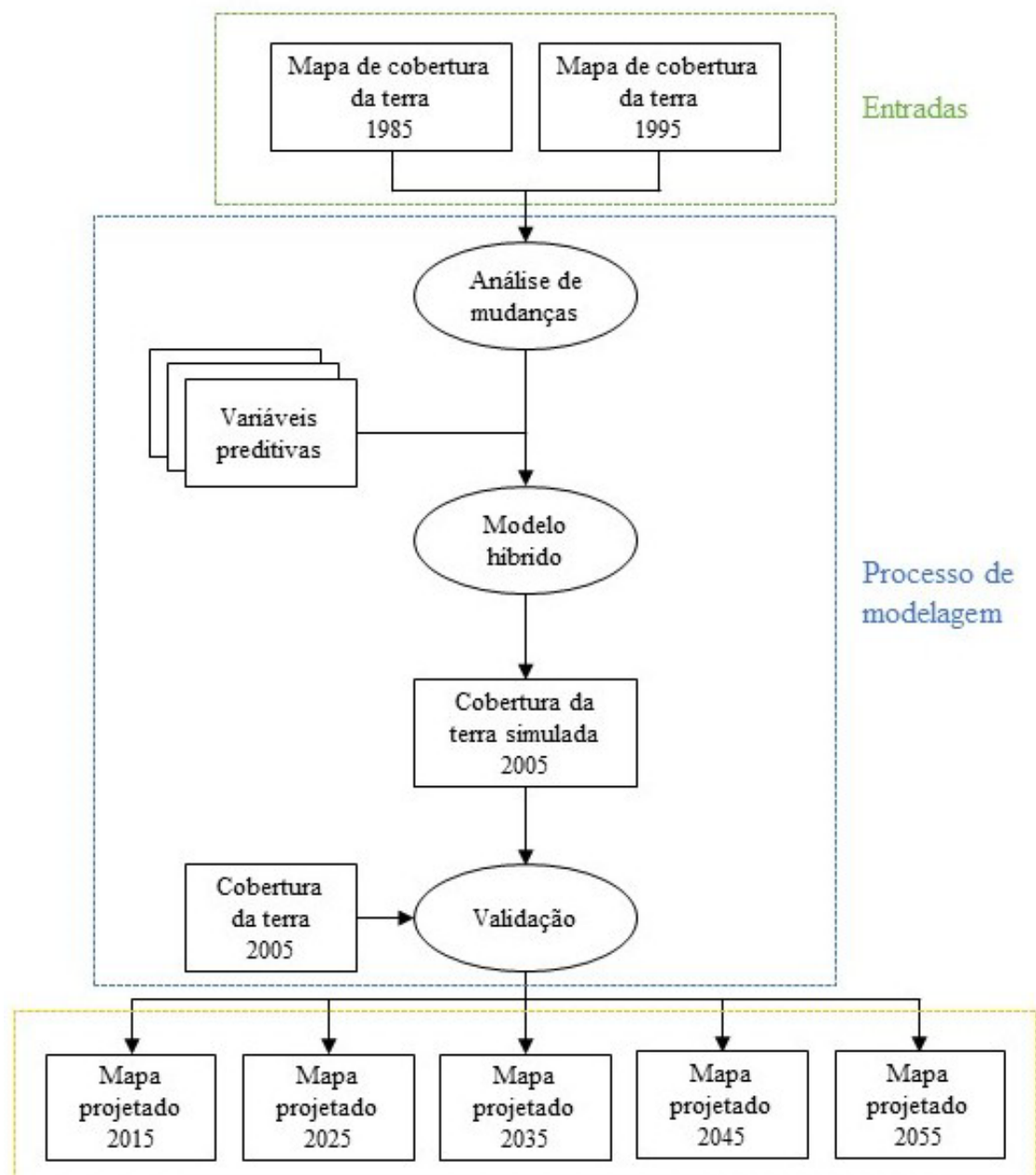

Figura 2. Fluxograma geral dos procedimentos da modelagem. Os retângulos indicam os mapas de entrada e saída e os círculos indicam os processos.

quase $80 \%$ das áreas úmidas em relação à extensão registrada em 1985, o que significa uma expansão de 132977 ha. Para resumir, até 2055 a área total de áreas úmidas abertas será equivalente a 300438 ha, com uma distribuição espacial significativamente orientada para a região central da área de estudo (Figura 3). 

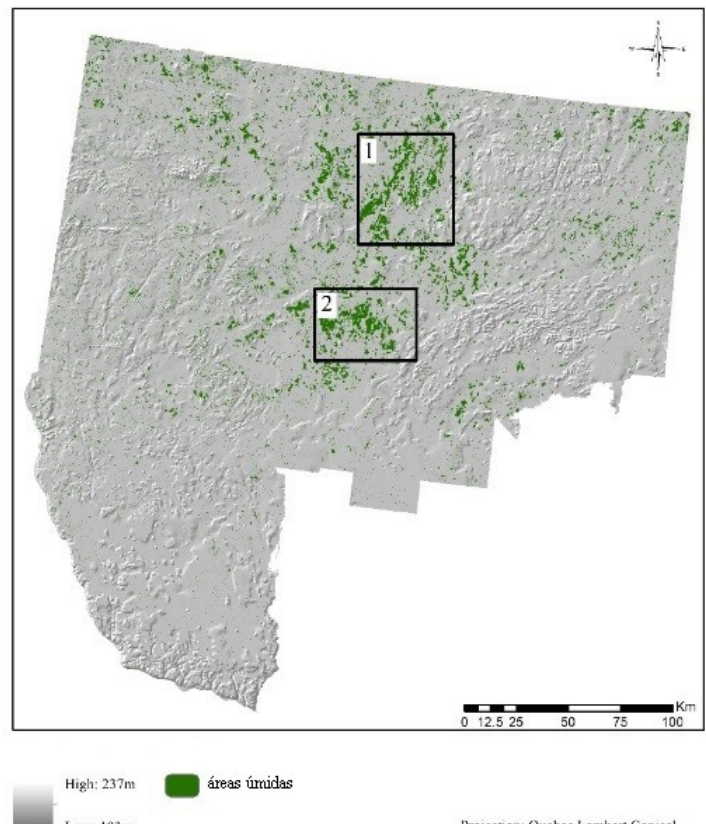

Low: $103 \mathrm{~m}$
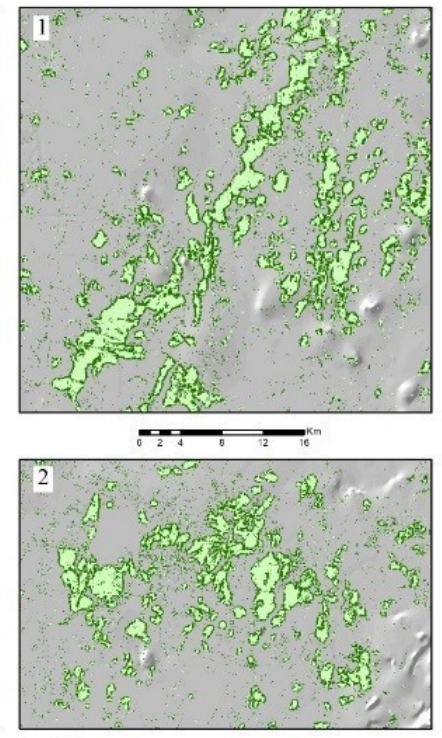

áreas úmidas - 1985

áreas úmidas - 2055

Figura 3. Distribuição das áreas úmidas observadas na imagem LANDSAT em 1985 e áreas simuladas em 2055 na região de estudo.

Dadas as características da região estudada, a abordagem híbrida usada neste estudo provou ser benéfica para entender a dinâmica complexa da distribuição de áreas úmidas abertas. Com o uso da regressão logística, as regras de transição do modelo foram automaticamente extraídas dos conjuntos de dados observados, em vez de serem determinadas a priori pelo desenvolvedor do modelo, como é usual na modelagem de AC. Estudos que realizaram a modelagem espaço-temporal de áreas úmidas foram desenvolvidos com sucesso, incluindo os que utilizaram modelos híbridos para analisar mudanças na paisagem, (Feng et al., 2016; Gong et al., 2014; Liu et al., 2015; Maeda et al., 2011; Mas et al., 2014; Ozah et al., 2010; Ozturk, 2015; Yu et al., 2010).

Ao estudar as áreas úmidas abertas do Québec, devemos levar em consideração alguns fatores. Em primeiro lugar, a distribuição e diversidade destas na zona boreal é definida principalmente por fatores físicos como geologia, topografia e clima, embora fatores biológicos e antrópicos também possam influenciar essa distribuição em menor proporção (Gingras et al., 2016). Em segundo lugar, a precisão dos dados utilizados pode ser crucial para os resultados. A maioria das áreas úmidas abertas na 
área de estudo são compostas por turfeiras (Lachance et al., 2016), difíceis de classificar usando apenas imagens do LANDSAT. Embora a faixa do infravermelho médio seja capaz de identificar corretamente as áreas úmidas (Leboeuf and Vaillancourt, 2015), a melhor opção seria, por conseguinte, combinar outros produtos de satélite e dados coletados no terreno.

Uma razão para diferença de tendência poderia ser o grande aumento de castores de 1980 a 2000 (Hood and Bayley, 2008; Lafond and Pilon, 2004; St-Pierre et al., 2017), quando a população pode ter dobrado (Lafond et al., 2003). Os castores são um agente conhecido de mudanças na dinâmica destes ecossistemas (St-Pierre et al., 2017; Touihri et al., 2018; Tremblay, 2009), ao construir as barragens, eles aprisionam a água, causando a morte de árvores e gerando novos locais de áreas úmidas, por outro lado, o abandono dessas barragens pode renovar a floresta da região anteriormente afetada. No entanto, as barragens de castores foram deixadas fora do modelo. Em geral o ciclo do castor (chegada e abandono do sítio) dura cerca de 30 anos (Lemelin and Darveau, 2008), dificultando a inclusão dessa dinâmica no modelo. Ao utilizar imagens de satélite, é quase impossível definir o momento do ciclo mencionado, desta forma, não há como projetar a evolução dessas barragens nas simulações.

Outra restrição é devido à escala usada. Como a região Abitibi-Témiscamingue é vasta, todas as mudanças são percebidas de maneira global, e pode haver áreas específicas com resultados diferentes quando uma escala menor é usada. A região de Abitibi-Témiscamingue é predominantemente plana, com um solo impermeável e drenagem deficiente, sendo estes os fatores fundamentais para o desenvolvimento de áreas úmidas (Gingras et al., 2016; Menard et al., 2013). Eles também são um resultado do processo de paludificação das florestas, que são influenciados por fatores físicos (topografia, geologia, clima), fatores biológicos (atividades do castor) e atividade humana (agricultura, silvicultura) (Lavoie et al., 2005). Como a região é pouco povoada, a manutenção desses sistemas é de certa forma preservada. Neste caso, o fator que mais ameaça esta área é o clima, especialmente para as turfeiras.

A influência das variações climáticas na distribuição de áreas úmidas, especificamente na zona boreal da área de estudo, foi outro fator ausente no desenho do modelo. A floresta boreal do Canadá enfrentará transformações importantes devido às mudanças climáticas (Nelson et al., 2014). As áreas úmidas abertas podem sofrer com o desequilíbrio hídrico causado pelas alterações climáticas, a menos que as perdas causadas pela evapotranspiração sejam compensadas pelo aumento das chuvas (Tarnocai, 2006). O desequilíbrio na disponibilidade de água em turfeiras arrisca os estoques de carbono acumulados há milhares de anos (Bridgham et al., 2006; Talbot et al., 2014), o que pode aumentar as emissões de dióxido de carbono na atmosfera, gerando repercussões bastante significativas no clima em escala planetária (Tarnocai, 2006). 
A região de Abitibi-Témiscamingue tem algumas particularidades que a tornam única. A baixa densidade populacional, juntamente com os esforços governamentais e privados de conservação, mantêm as áreas úmidas estáveis (Gingras et al., 2016). Os resultados deste estudo servem como base para entender melhor a probabilidade de mudanças em áreas úmidas abertas e para apoiar os processos de tomada de decisão, a fim de evitar grandes impactos nesses ecossistemas.

\section{Conclusão}

O objetivo deste estudo foi desenvolver um modelo capaz de simular cenários futuros das áreas úmidas abertas da região de Abitibi-Témiscamingue no Québec, Canadá. A abordagem metodológica utilizada foi a de um modelo híbrido que provou ser uma ferramenta eficiente para estudar as mudanças na cobertura da terra, o que o torna um instrumento eficaz na projeção de cenários futuros. As projeções foram validadas com sucesso por métodos estatísticos de concordância e discordância espacial e quantitativa.

Devido às limitações de dados, não foi possível incluir no modelo importantes fatores de mudança de áreas úmidas abertas. Embora os resultados da simulação tenham mostrado uma tendência crescente na superfície destas áreas, ainda há considerações adicionais a serem levadas em conta em futuros estudos. Por exemplo, o acesso à melhor resolução espacial em produtos de sensoriamento remoto fornecerá informações mais detalhadas sobre diferentes categorias ou tipos de áreas úmidas que não foram detectadas através do LANDSAT. Além disso, a mudança climática ainda permanece como um fator incerto ligado às estes ecossistemas e ainda não está claro como eles podem reagir ao aquecimento global. Portanto, outros esforços de simulação serão muito beneficiados com a inclusão de variáveis climáticas para entender claramente a dinâmica entre estas e a distribuição espacial de áreas úmidas na região, obtendo respostas cada vez mais precisas aos mais variados cenários futuros.

\section{Bibliografia}

Arsanjani, J.J., Helbich, M., Kainz, W., Boloorani, A.D. (2012). "Integration of logistic regression, Markov chain and cellular automata models to simulate urban expansion", Int. J. Appl. Earth Obs. Geoinf., 21: 265-275.

Arsanjani, T.J., Javidan, R., Nazemosadat, M.J., Arsanjani, J.J., Vaz, E. (2015). “Spatiotemporal monitoring of Bakhtegan Lake's areal fluctuations and an exploration of its future status by applying a cellular automata model", Comput. Geosci., 78: 37-43. 
Batty, M. (2012). “A Generic Framework for Computational Spatial Modelling”, in Heppenstall, A. (ed.), Agent-Based Models of Geographical Systems, Springer Science, pp. 19-32.

Bridgham, S.D., Megonigal, J.P., Keller, J.K., Bliss, N.B. \& Trettin, C. (2006). “The carbon balance of North American wetlands", Wetlands 26: 889-916.

Chen, S.H., Jakeman, A.J. \& Norton, J.P. (2008). “Artificial Intelligence techniques: An introduction to their use for modelling environmental systems", Math. Comput. Simul., 78: 379-400.

Eamer, J., Hayes, T., Simth, R. (2010). Canadian Biodiversity: Ecosystem Status and Trends 2010, Federal, Provincial, and Territorial Governments of Canada.

Eastman, J.R., 2015. TerrSet: Geospatial Monitoring and Modeling Software, Clark Labs. Clark University, Worcester.

Efron, B. (1988). "Logistic Regression, Survival Analysis, and the Kaplan-Meier Curve", J. Am. Stat. Assoc., 83: 414-425.

Environment and Climate Change Canada (2016). Canadian Environmental Sustainability Indicators: Extent of Canada's Wetlands, Gatineau, QC.

Feng, Y., Yang, Q., Hong, Z., Cui, L. (2016). "Modelling coastal land use change by incorporating spatial autocorrelation into cellular automata models", Geocarto Int., 6049: 1-19.

Frolking, S., Talbot, J., Jones, M.C., Treat, C.C., Kauffman, J.B., Tuittila, E.-S., Roulet, N. (2011). "Peatlands in the Earth's 21st century climate system", Environ. Rev., 19: 371-396.

Gingras, B., Slattery, S., Smith, K., Darveau, M.(2016). Boreal Wetlands of Canada and the United States of America, pp. 1-23.

Gong, W., Yuan, L., Fan, W., Stott, P. (2014). “Analysis and simulation of land use spatial pattern in Harbin prefecture based on trajectories and cellular automata Markov modelling", Int. J. Appl. Earth Obs. Geoinf., 34: 207-216.

Hattermann, F.F., Krysanova, V., Hesse, C. (2008). "Modelling wetland processes in regional applications", Hydrol. Sci. J., 53: 1001-1012.

Hood, G.A., Bayley, S.E. (2008). "Beaver (Castor canadensis) mitigate the effects of climate on the area of open water in boreal wetlands in western Canada", Biol. Conserv., 141: 556-567.

Hyandye, C., Martz, L.W. (2017). “A Markovian and cellular automata land-use change predictive model of the Usangu Catchment.", Int. J. Remote Sens., 38: 6481 .

Kottek, M., Grieser, J., Beck, C., Rudolf, B., Rubel, F. (2006). "World map of the Köppen-Geiger climate classification updated”, Meteorol. Zeitschrift, 15: 259263. 
Kumar, K.S., Kumari, K.P., Bhaskar, P.U. (2016). “Application of Markov Chain \& Cellular Automata based model for prediction of Urban transitions", Int. Conf. Electr. Electron. Optim. Tech. - 2016 4007-4012.

Lachance, S.-C., Lapointe, M., Darveau, M., Imbeau, L. (2016). Méthode de classification des milieux humides du Québec boréal à partir de la carte écoforestière du 4e inventaire décennal, Québec.

Lafond, R., Pilon, C. (2004). "Abondance du castor (Castor canadensis) au Québec. Bilan d 'un programme d 'inventaire aérien”, Le Nat. Can. - Faune, 128: 43-51.

Lafond, R., Pilon, C., Leblanc, Y. (2003). Bilan du plan d'inventaire aérien des colonies de castors au Québec (1989-1994), Societé de la faune et des Parcs du Québec, Québec.

Langlois, P. (2008) Cellular automata for modeling spatial systems. Model. Process Geogr. From Detreminism to Complex, 278-306 pp.

Lavoie, M., Paré, D., Fenton, N., Groot, A., Taylor, K. (2005). "Paludification and management of forested peatlands in Canada: a literature review", Environ. Rev., 13: 21-50.

Leboeuf, A., Vaillancourt, E. (2015). Guide de photo-interprétation des essences forestières du Québec méridional, Gouvernement du Québec, Québec.

Lemelin, L.-V., Darveau, M. (2008). "Les milieux humides du parc national du Canada de la Mauricie: cartographie en vue d'une surveillance de l'intégrité écologique", Rapport technique $n^{\circ}$ Q11, Canards Illimités Canada, Québec.

Lemelin, L.V., Darveau, M., Imbeau, L., Bordage, D. (2010). "Wetland Use and Selection by Breeding Waterbirds in the Boreal Forest of Quebec, Canada", Soc. Wetl. Sci., 30: 321-332.

Lin, Y.-P., Chu, H.-J., Wu, C.-F., Verburg, P.H. (2011). "Predictive ability of logistic regression, auto-logistic regression and neural network models in empirical landuse change modeling - a case study", Int. J. Geogr. Inf. Sci., 25: 65-87.

Liu, Y., Dai, L., Xiong, H. (2015). "Simulation of urban expansion patterns by integrating auto-logistic regression, Markov chain and cellular automata models", J. Environ. Plan. Manag., 58: 1113-1136.

Luo, Geping, Tureniguli Amuti, B., Lei Zhu, B., Bulkajyr Mambetov, B.T., Bagila Maisupova, B., Chi Zhang, B., Ignacio Lopez Moreno, J., Luo, G, Amuti Á C Zhang, Á.T., Amuti, T., Zhang, C., Zhu, L., Mambetov Á B Maisupova, B.T. (2015) Dynamics of landscape patterns in an inland river delta of Central Asia based on a cellular automata-Markov model. Reg Env. Chang. 15, 277-289.

Maeda, E.E., de Almeida, C.M., de Carvalho Ximenes A., A., Formaggio, A.R., Shimabukuro, Y.E., Pellikka, P. (2011). "Dynamic modeling of forest conversion: Simulation of past and future scenarios of rural activities expansion in the fringes of the Xingu National Park, Brazilian Amazon”, Int. J. Appl. Earth Obs. Geoinf., 13: 435-446. 
Mas, J.-F., Kolb, M., Paegelow, M., Camacho Olmedo, M.T., Houet, T. (2014). “Inductive pattern-based land use/cover change models: A comparison of four software packages", Environ. Model. Softw., 51: 94-111.

Menard, S., Darveau, M., Imbeau, L. (2013). "The importance of geology, climate and anthropogenic disturbance sin shaping boreal wetland and aquatic landscape types", Ecoscience, 20: 399-410.

Ménard, S., Darveau, M., Imbeau, L. (2007). "Histoire de la classification des milieux humides au Québec", Le Nat. Can., 131: 85-90.

Nelson, T.A., Coops, N.C., Wulder, M.A., Perez, L., Fitterer, J., Powers, R., Fontana, F. (2014). "Predicting climate change impacts to the canadian boreal forest", Diversity, 6: 133-157.

Ozah, A.P., Adesina, F.A., Dami, A. (2010). "A Deterministic Cellular Automata Model for Simulating Rural Land Use Dynamics: A Case Study of Lake Chad Basin”, ISPRS Arch. XXXVIII, pp. 75-81.

Ozturk, D. (2015). "Urban Growth Simulation of Atakum (Samsun, Turkey) Using Cellular Automata-Markov Chain and Multi-Layer Perceptron-Markov Chain Models", Remote Sens. , 7: 5918-5950.

Pellerin, S., Poulin, M. (2013). Analyse de la situation des milieux humides au Québec et recommandations à des fins de conservation et de gestion durable, Ministère du Développement durable, de l'Environnement, de la Faune et des Parcs.

Pontius, R. (2000). "Quantification error versus location error in comparison of categorical maps", Photogramm. Eng. Remote Sensing.

Pontius, R.G., Millones, M. (2011). "Death to Kappa: birth of quantity disagreement and allocation disagreement for accuracy assessment", Int. J. Remote Sens., 32: 4407-4429.

Poulin, M., Pellerin, S., Jérôme Cimon-MorinSophie Lavallée, G., Youri, C., Youri, T. (2016). "Inefficacy of wetland legislation for conserving Quebec wetlands as revealed by mapping of recent disturbances", Wetl. Ecol. Manag., 24: 651-665.

Qiang, Y., Lam, N.S.N. (2015). "Modeling land use and land cover changes in a vulnerable coastal region using artificial neural networks and cellular automata", Environ. Monit. Assess., 187: 94-111.

Ramsar, S. de la C. de (2010). Utilisation rationnelle et conservation des zones humides, $4 \mathrm{e}$ ed. Secrétariat de la Convention de Ramsar, Gland.

St-Pierre, M.L., Labbé, J., Darveau, M., Imbeau, L., Mazerolle, M.J. (2017). "Factors Affecting Abundance of Beaver Dams in Forested Landscapes", Wetlands, 37: 941-949.

Talbot, J., Roulet, N.T., Sonnentag, O., Moore, T.R. (2014). "Increases in aboveground biomass and leaf area 85 years after drainage in a bog", Botany, 92 : 713-721. 
Tarnocai, C. (2006). "The effect of climate change on carbon in Canadian peatlands", Glob. Planet. Change, 53: 222-232.

Touihri, M., Labbé, J., Imbeau, L., Darveau, M. (2018). "North American Beaver (Castor canadensis Kuhl) Key Habitat Characteristics: Review of the Relative Effects of Geomorphology", Food Availability and Anthropogenic Infrastructure. Ecoscience, 25: 9-23.

Tremblay, G. (2009). Caractérisation des paramètres de l'habitat du castor qui favorisent l'utilisation des ponceaux comme site de construction de barrage. Université du Québec en Abitibi-Témiscamingue.

Valeria, O., Laamrani, A., Beaudoin, A. (2014). "Monitoring the state of a large boreal forest region in eastern Canada through the use of multitemporal classified satellite imagery", Can. J. Remote Sens., 38: 91-108.

Warner, B., Rubec, C. (1997). Système de classification des terres humides du Canada, 2nd ed, Centre de recherche sur les terres humides, Université de Waterloo, Waterloo.

Yu, H., He, Z., Pan, X. (2010). "Wetlands shrink simulation using Cellular Automata: A case study in Sanjiang Plain, China”, Procedia Environ. Sci., 2: 225-233.

Zedler, J.B., Kercher, S. (2005). "Wetland Resources: Status, Trends, Ecosystem Services, and Restorability”, Annu. Rev. Environ. Resour., 30: 39-74.

Zhu, P., Gong, P. (2014). "Suitability mapping of global wetland areas and validation with remotely sensed data", Sci. China-Earth Sci., 57: 2283-2292. 